10.1515/astro-2017-0390

Baltic Astronomy, vol.21, 319-330, 2012

\title{
CROSS CATALOGUE MATCHING WITH VIRTUAL OBSERVATORY AND PARAMETERIZATION OF STARS
}

O. Malkov ${ }^{1,2}$, O. Dluzhnevskaya ${ }^{1}$, S. Karpov ${ }^{3}$, E. Kilpio ${ }^{1}$, A. Kniazev ${ }^{4,5}$, A. Mironov ${ }^{5}$ and S. Sichevskij ${ }^{1}$

1 Institute of Astronomy, Russian Acad. Sci., 48 Pyatnitskaya, Moscow 119017, Russia;malkov@inasan.ru

2 Faculty of Physics, Lomonosov Moscow State University, Leninskie Gory, Moscow 119991 GSP-1, Russia;

3 Special Astrophysical Observatory, Russian Acad. Sci., Nizhnij Arkhyz, Zelenchukskiy region, Karachai-Cherkessian Republic, 369167, Russia; karpov@sao.ru

4 South African Astronomical Observatory, PO Box 9, Observatory 7935, South Africa; akniazev@saao.ac.za

5 Sternberg Astronomical Institute, Lomonosov Moscow State University, 13 Universitetskij Prosp., Moscow 119992, Russia; almir@sai.msu.ru

Received: 2012 February 13; accepted: 2012 May 18

\begin{abstract}
Virtual Observatory facilities allow users to make a fast and correct cross-matching of objects from various surveys. They yield multi-color photometry data (color indices) on registered objects and make it possible to determine stellar parameters. A method of catalogue cross-matching, as well as its application to various areas in the sky and preliminary results of stellar parameterization, are discussed in the paper. We also make a critical analysis of modern stellar spectral atlases.
\end{abstract}

Key words: stars: fundamental parameters - photometric surveys - crossmatching - virtual observatory - spectral atlases - unresolved binaries

\section{INTRODUCTION}

Color index diagrams can serve as powerful tools for determination of stellar intrinsic energy distribution and, hence, for parameterization of stars. This is tightly related to the study of interstellar extinction because the spectral energy distribution can be examined only once the observed spectra are corrected for interstellar reddening.

Recent large photometric surveys as well as tools for cross-matching their objects provide us, in principle, with a possibility to get multi-color photometric data for millions of objects. Consequently, they allow user to construct color-index diagrams for classification and parameterization of objects. To correctly determine stellar parameters from observed photometry, response curves of analyzed surveys, 

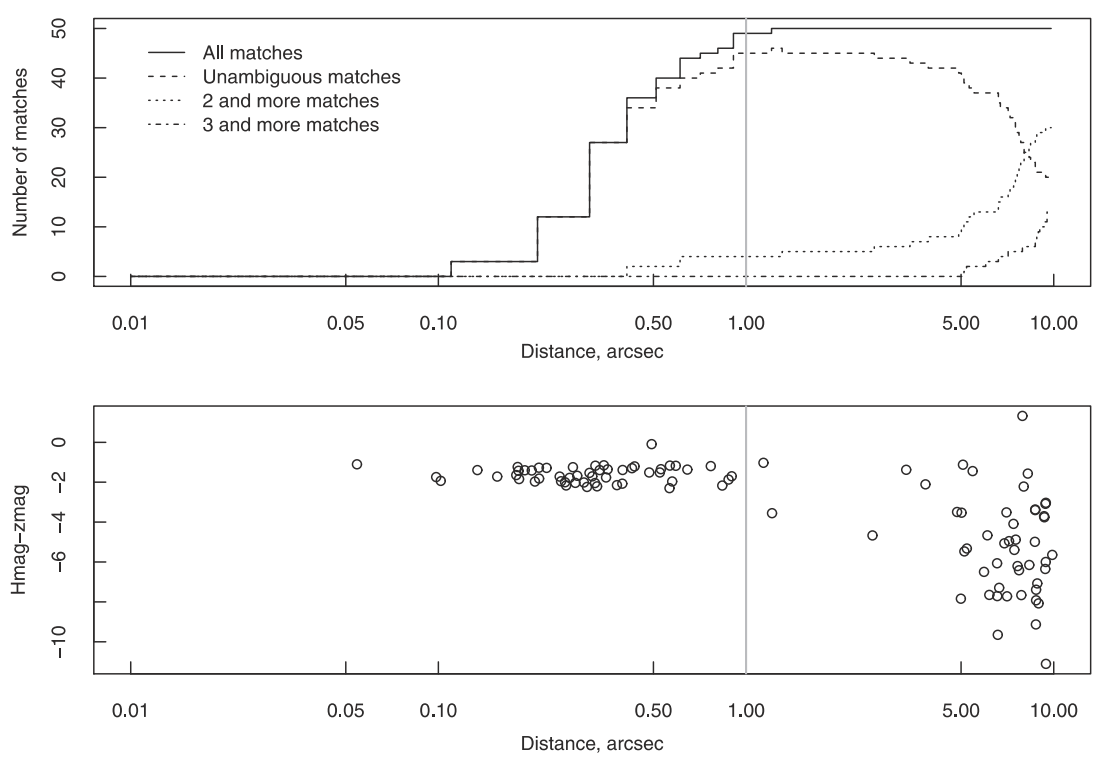

Fig. 1. Upper panel: a typical dependence of the number of matches on the match radius for a small overlapping region of $2 \mathrm{MASS}$ and SDSS. Lower panel: the scatter plot of a color-magnitude difference between measurements in nearby spectral bands of two catalogues for matches as a function of distance. The vertical line marks an optimal match radius.

interstellar extinction law, and empirical spectral energy distributions for stars of different spectral types should be known.

Cross-matching of catalogues is described in Section 2. Construction of color index diagrams and parameterization of objects are discussed in Section 3. Section 4 contains the results of our critical analysis of modern stellar spectral atlases. In Section 5, we draw our conclusions.

\section{CROSS-CORRELATION OF CATALOGUES}

The task of catalogue cross-matching is to reliably link the occurrences of the same astrophysical source in different catalogues, which may be acquired from observations on different epochs, with different instruments, in various spectral filters, etc. Typical problems that arise here include bad coordinate accuracy of one or several catalogues, possible proper motions for nearby objects, and different sensitivities of the surveys, which may lead to significantly varying object densities. Solely positional information is, therefore, insufficient to perform a reliable match: the "true" object may, by chance, be at a larger distance from the expected position than some other one.

A possible solution is to use some prior information when comparing nearby records from different catalogues. If they have measurements in the same photometric filter, one may select only objects with roughly the same magnitudes; if magnitudes do not match, spectral shape information may be used. Indeed, object colors - or the slope of the spectrum - for similar objects are also similar, and, for some region, reliably matched points will be clustered not only in inter-point distance but in colors (computed using magnitudes from different catalogues) too. 




Fig. 2. Response curves of the analyzed catalogues.

Finding such clusters and performing iterative sigma-clipping filtering, one may choose appropriate limits for match radius and magnitude difference. Figure 1 illustrates this concept.

It can be seen from Fig. 1 that one arcsecond is a good approximation for the match radius for the $2 \mathrm{MASS}$ and SDSS catalogues. Our analysis shows that it is true for any pair of the catalogues (all analyzed catalogues are listed in Table and their response curves are presented in Fig. 2) and any galactic latitude. An exception is GALEX, and we have chosen a value of three arcseconds for the matching radius if a pair of matched catalogues contains GALEX. Budavari et al. (2009), cross-identifying GALEX with SDSS, note that FUV and NUV sources at smaller separations are already merged in GALEX.

Also, the consequent use of matched objects in spectral modeling and determination of extinction provides one more chance to filter out possible random coincidences by excluding objects giving results significantly different from those for the ensemble.

We have developed an algorithm of fast positional matching of large astronomical catalogues in small (up to one degree) areas with false-solution filtering. Informational technologies we have used for cross-matching in our work are de- 
Table 1. Large photometric catalogues.

\begin{tabular}{|c|c|c|c|c|}
\hline $\begin{array}{l}\text { Catalogue, } \\
\text { reference }\end{array}$ & $\begin{array}{r}\text { Number of } \\
\text { objects }\end{array}$ & $\begin{array}{r}\text { Sky } \\
\text { coverage }\end{array}$ & $\begin{array}{r}\text { Photometric } \\
\text { bands }\end{array}$ & $\begin{array}{r}\text { Magnitude } \\
\text { limit }\end{array}$ \\
\hline DENIS & $\begin{array}{c}355 \mathrm{mln} \\
\text { u-strasbg.fr }\end{array}$ & $\begin{array}{r}50 \% \\
\text { lenis.htm }\end{array}$ & Gunn- $i, J, K_{S}$ & $18.5,16.5,14.0$ \\
\hline 2MASS & $\begin{array}{l}300 \mathrm{mln} \\
\text { pac.caltech.ed }\end{array}$ & $\begin{array}{r}100 \% \\
\text { / } 2 \text { mass }\end{array}$ & $J, H, K_{S}$ & $15.8,15.1,14.3$ \\
\hline SDSS & $500 \mathrm{mln}$ & $\begin{array}{r}25 \% \\
\text { sdss.org/ }\end{array}$ & $u, g, r, i, z$ & $g, r=22.2$ \\
\hline GALEX (AIS) & $\begin{array}{r}90 \mathrm{mln} \\
\text { www.galex.ca }\end{array}$ & $\begin{array}{r}90 \% \\
\text { tech.edu/ }\end{array}$ & FUV, NUV & $\sim 25$ \\
\hline $\begin{array}{l}\text { UKIDSS } \\
\text { (LAS+GPS) }\end{array}$ & $\begin{array}{l}40 \mathrm{mln} \\
\text { www.u }\end{array}$ & $\begin{array}{r}15 \% \\
\text { ridss.org/ }\end{array}$ & $Z, Y, J, H, K$ & $K=18.3$ \\
\hline
\end{tabular}

scribed in Malkov \& Karpov (2011). To test the tool, it was applied to seven 0.1-degree areas, where objects from DENIS, 2MASS, SDSS, GALEX, UKIDSS surveys were selected and cross-matched. The corresponding sub-catalogues were compiled in the VOTable format (Karpov et al. 2012). Our results show that the constructed procedure can be used for cross-matching of objects in arbitrary areas of the sky for consequent parameterization of stars and determination of interstellar extinction in the Galaxy.

\section{COLOR INDEX DIAGRAMS}

\subsection{Construction of color index diagrams}

The cross-matched stars can be plotted on combined color index diagrams. To determine their stellar intrinsic energy distribution and, hence, stellar parameters, one should compare observed and theoretical color indices.

We show theoretical and observational points in the FUV-NUV $-u-g$ plane, where $u, g$ are SDSS photometric bands and FUV, NUV, are GALEX photometric bands, in Fig. 3 To simulate stars in the diagram, we used the spectral atlas by Pickles (1998) (see criticism of spectral atlases in Section 4) and the interstellar extinction law from Fluks et al. (1994). The SDSS and GALEX response curves were taken from Gunn et al. (1998) and Morrissey et al. (2005), respectively (see Fig. 2).

There exist several reasons for the observed disagreement between the empirical and theoretical points in Fig. 3: observational photometric uncertainties, misprints in the catalogues, cross-match errors, variability or non-stellar nature of objects, non-standard behavior of the interstellar extinction law in the area, etc. One of possible reasons are unresolved binaries: close binaries, which can be resolved neither photometrically (unless they exhibit mutual eclipses) nor astrometrically.

\subsection{Recognition of binaries in color-index diagrams}

Unresolved binaries with components of different temperatures can exhibit colors different enough from those of single stars. Such binaries can be separated from single stars in some color-index diagrams. In Malkov et al. (2011), we have shown 


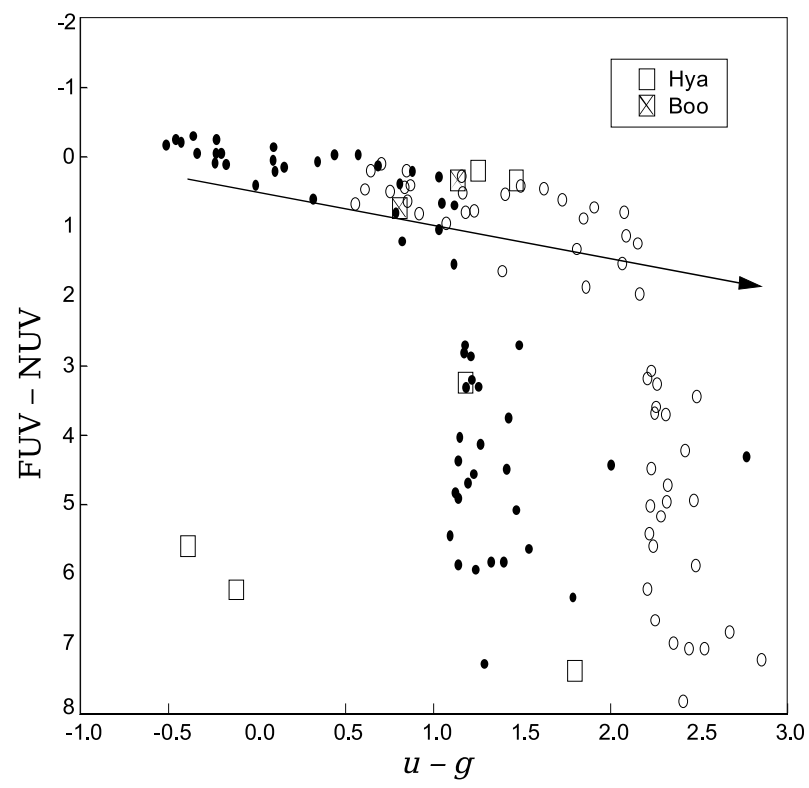

Fig. 3. SDSS/GALEX photometry. Squares: SDSS/GALEX objects in Bootes $(l=$ $353^{\circ}, b=+68^{\circ}, r=0.1^{\circ}$, crossed squares $)$ and Hydra $\left(l=228^{\circ}, b=+27^{\circ}, r=0.1^{\circ}\right.$, open squares) areas. Filled circles are Pickles (1998) models recalculated into the AB magnitude system. Open circles are the same models but reddened by $E_{B-V}=1$. The arrow is the reddening line.

that GALEX/SDSS photometry and Gaia photometry can be used for singlebinary star separation and for parameterization of stars. Particularly, we have found Gaia color indices most suitable for single-binary star separation.

Obviously, the interstellar reddening complicates identification of unresolved binaries in color-index diagrams. However, for some binaries, color indices can be indicated where interstellar reddening does not prevent discovery of the pair.

\subsection{Parametrization of stars}

Cross-matching objects in large catalogues provides us with multi-color photometry, which can be used for parameterization of stars. We have developed a method of obtaining the most reliable spectral type of a star from available observational photometry and data from one of atlases of spectral energy distributions. Varying models of stellar spectrum in the atlas, one can select the "best" spectral type and color excess $E_{B-V}$, so that the residual (i.e. the difference between the observed data and those from the atlas) reaches its minimum. As an example, the residuals for the star HD 1796 (K3III) are shown in Fig. 4 as a function of model spectral type. The method also allows to estimate the distance to the star and interstellar extinction.

Approbation of the method using bright stars with known spectral classification and $W B V R$ photometry has allowed us to estimate reliability of our method of classification of stars. We have used about seventy spectrum models (luminosity classes I, III. and V), collected in the Pickles (1998) atlas, and the interstellar extinction law by Fluks et al. (1994). A detailed descruption of the method and the 


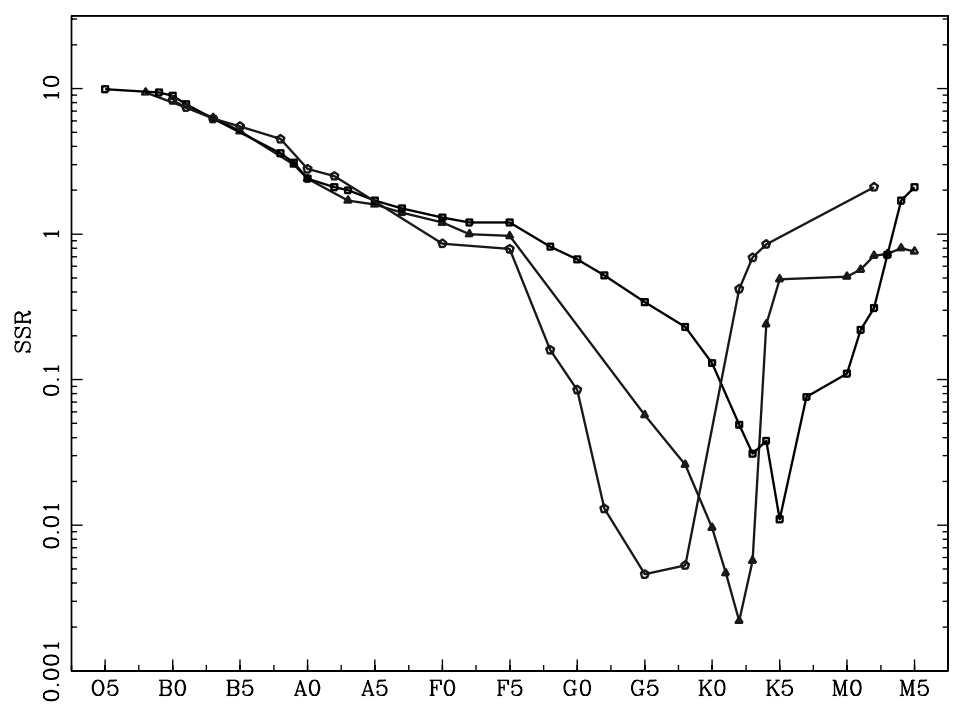

Fig. 4. The sum of squared residuals obtained for the star HD 1796 (K3III) vs. the model spectral type. The circles, triangles, and squares indicate supergiants, giants, and dwarfs, respectively.

parameterization results are given in Sichevskij (2012) and Sichevskij et al. (2012).

Thus, multi-color photometry and spectral classification provide us with stellar temperatures and luminosities. Then, a method for estimating stellar masses and ages, based on the geometric similarity of evolutionary tracks for stars at the same evolutionary stage in the HR diagram (Malkov et al. 2010), can be applied.

\section{CRITICISM OF STELLAR SPECTRAL ATLASES}

For a great variety of astrophysical applications, it is extremely important to know (at least a relative) SED for (i) as many stars as possible and (ii) as many types of stars as possible. Semi-empirical (or combined) spectrophotometric atlases are designed to meet the latter requirement, while to satisfy the former one, a large number of empirical atlases are constructed.

\subsection{Semi-empirical spectral atlases}

The Asiago Database of Spectroscopic Databases (Sordo \& Munari, 2006) contains several semi-empirical atlases. Only two of them cover a rather wide spectral range: Sviderskiene (1988) (1200-10500 A) and Pickles (1998) (1150-25000 Å). As mentioned in Section 3, we have used the semi-empirical stellar spectral atlas by Pickles (1998) to simulate stars on color-color diagrams. It is widely used and remains one of the best spectral atlases; however, as our analysis shows, synthetic magnitudes calculated with Pickles data appreciably differ from observed ones for some types of stars. Particularly (see Fig. 5), $B, V, R$ magnitudes for $\mathrm{K} 4$ and later giants predicted by Pickles (1998) deviate significantly from those listed in the $W B V R$ catalogue (Kornilov et al. 1991).

It should be noted here that $\mathrm{M}$ giants can serve as exceptionally useful sources for determination of stellar parameters and extinction values. It was shown by 


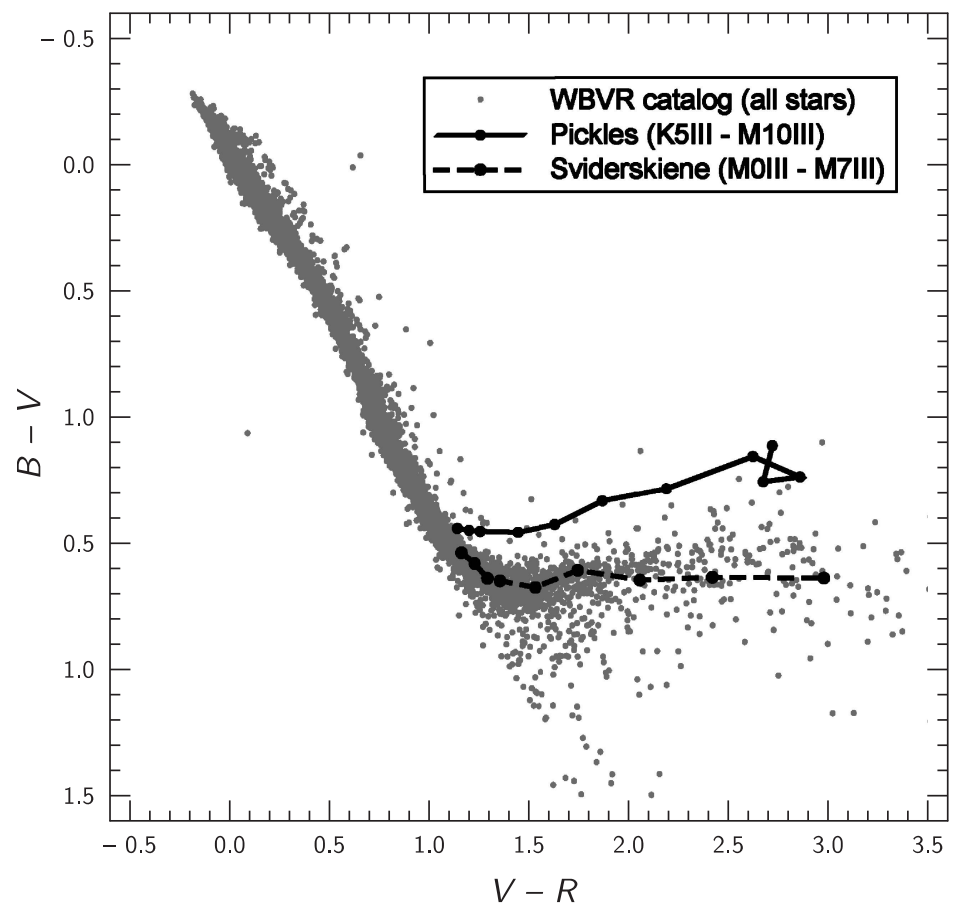

Fig. 5. Synthetic $B, V, R$ photometry, calculated from Pickles (1998) and Sviderskiene (1988) data for red giants, together with observational data from the $W B V R$ catalogue (Kornilov et al. 1991).

Mironov et al. (2010) that, in the $(B-V, V-I)$ two color diagram, the angle between the intrinsic color line for M4-M8 giants and that for other stars is significant (cf. Fig. 5). Consequently, the reddening curves also form a significant angle with the M-giant intrinsic color line. Thus, observational colors can be relatively easily "de-reddened", and stellar parameters, including extinction, can be estimated.

It should be also noted (see Fig. 6) that data for O-type stars in the Pickles (1998) atlas were not corrected for interstellar extinction carefully or specifically enough. The Pickles curve reproduces a SED for the faint, presumably reddened HD 48279, while data from Sviderskiene (1988) show a good agreement with the brighter, presumably unreddened HD 47839 .

\subsection{Empirical spectral atlases}

Data from empirical stellar spectral atlases can also be used to construct colorindex diagrams. Early empirical atlases (e.g., Breger 1976, Gunn and Stryker 1983) contained several hundred stars. In the late 1980s, more representative atlases took their place: The Spectrophotometric Catalogue of Stars (hereafter SCS) by Kharitonov et al. (1988), the Sternberg Spectrophotometric Catalogue by Glushneva et al. (1982-1984) with its IR extention, the Moscow Spectrophotometric Catalogue by Glushneva et al. (1980-1991). Most precise data on 238 secondary spectrophotometric standards (hereafter SSS) were collected by Glushneva et al. (1992). 


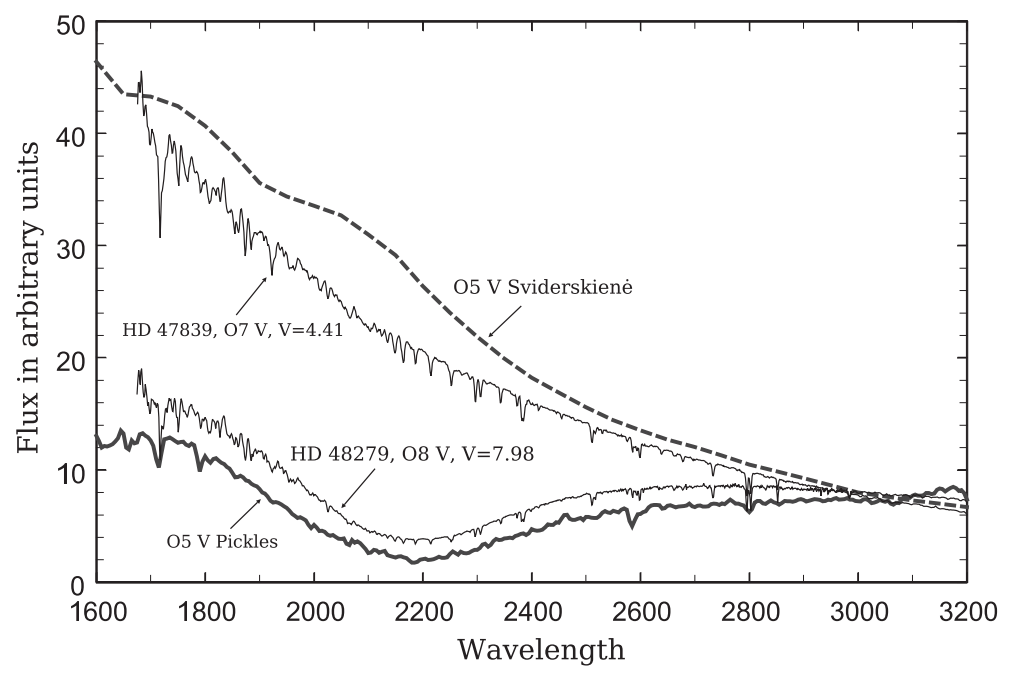

Fig. 6. O stars: observational data and semi-empirical atlases by Sviderskiene (1988) and Pickles (1998). See explanation in the text.

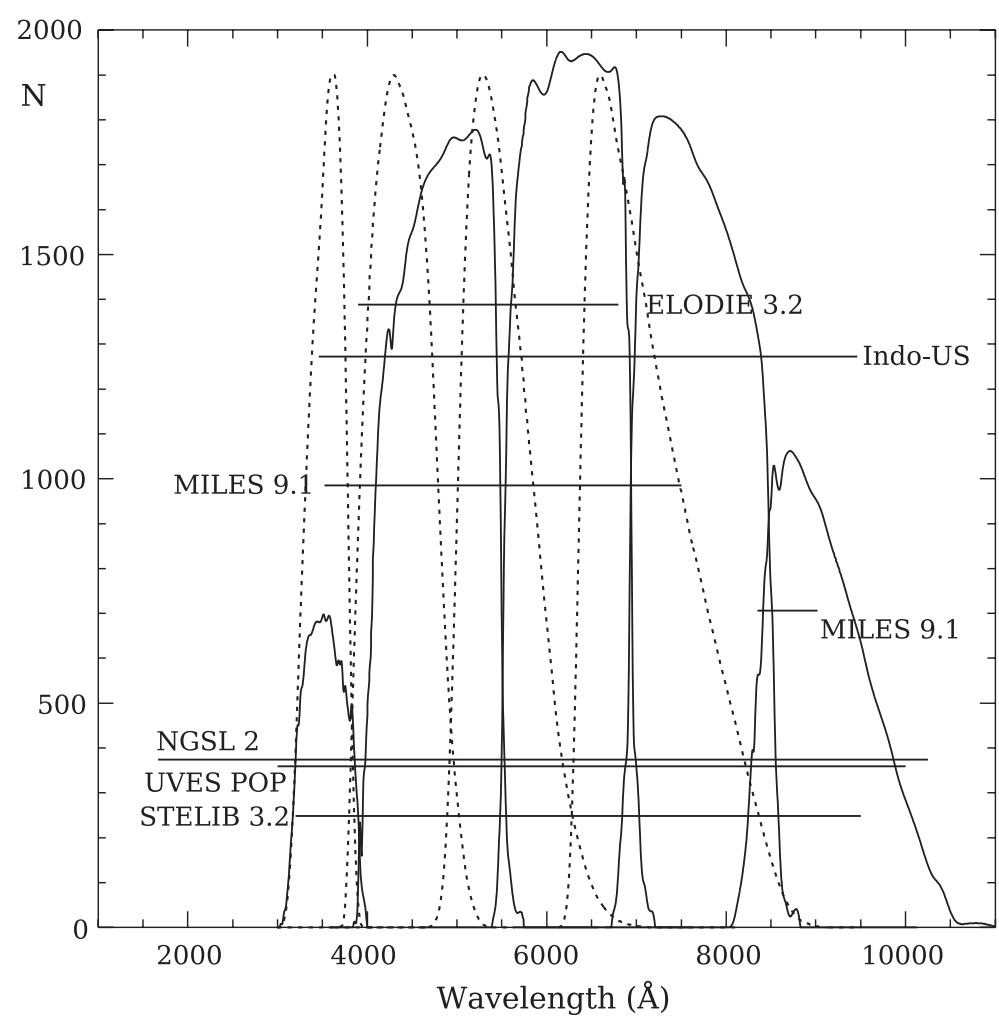

Fig. 7. The horizontal lines represent modern empirical spectrophotometric atlases: number of stars vs. spectral coverage. The SDSS (solid) and $W B V R$ (dashed) response curves are also shown. 
Table 2. Empirical spectral atlases.

\begin{tabular}{|c|c|c|c|}
\hline Name & $\begin{array}{l}\text { Number } \\
\text { of stars }\end{array}$ & $\begin{array}{l}\text { Spectral } \\
\text { range, } \AA\end{array}$ & Reference \\
\hline ELODIE 3.2 & 1388 & $3900-6800$ & Wu et al. (2011) \\
\hline \multicolumn{4}{|c|}{ http://www.obs.u-bordeaux1.fr/m2a/soubiran/elodie_library.html } \\
\hline Indo-US / CFLIB & $1273^{1}$ & $3460-9464$ & Valdes et al. (2004) \\
\hline \multicolumn{4}{|c|}{ http://www.noao.edu/cflib/ } \\
\hline \multirow[t]{2}{*}{ MILES 9.1} & 985 & $3525-7500$ & Falcon-Barroso et al. (2011) \\
\hline & 706 & $8350-9020$ & \\
\hline \multicolumn{4}{|l|}{ http://miles.iac.es } \\
\hline NGSL 2 & 374 & $1670-10250$ & Heap and Lindler (2007) \\
\hline \multicolumn{4}{|c|}{ http://archive.stsci.edu/prepds/stisngsl/ } \\
\hline STELIB 3.2 & 249 & $3200-9500$ & Le Borgne et al. (2003) \\
\hline \multicolumn{4}{|c|}{ http://webast.ast.obs-mip.fr/stelib } \\
\hline UVES POP & $359^{2}$ & $3000-10000$ & Bagnulo et al. (2003) \\
\hline \multicolumn{4}{|c|}{ http://www.sc.eso.org/santiago/uvespop/ } \\
\hline SCS & 1147 & $3225-7575$ & Kharitonov et al. $(1988,2011)$ \\
\hline \multicolumn{4}{|l|}{ VizieR: III/202 } \\
\hline \multirow[t]{2}{*}{ SSS } & 238 & $3200-7600$ & Glushneva et al. (1992) \\
\hline & $99^{3}$ & $6000-10800$ & \\
\hline \multicolumn{4}{|c|}{ VizieR: J/A+AS/92/1 } \\
\hline \multirow[t]{2}{*}{ Pulkovo } & 679 & $3200-7350$ & Alekseeva et al. $(1996,1997)$ \\
\hline & $278^{3}$ & $3200-10800$ & \\
\hline \multicolumn{4}{|l|}{ VizieR: III/201 } \\
\hline
\end{tabular}

Table 3. Comparison of synthetic and catalogued (Kornilov et al. 1991) colors

\begin{tabular}{l|rrrrrr}
\hline & UVES-POP & STELIB & MILES & Indo-US & NGSL & SSS \\
\hline$\sigma_{W-B}$ & 0.043 & 0.118 & 0.192 & 0.210 & 0.028 & 0.072 \\
No. of stars & 7 & 13 & 34 & 76 & 15 & 231 \\
\hline$\sigma_{B-V}$ & 0.015 & 0.093 & 0.113 & 0.087 & 0.018 & 0.020 \\
No. of stars & 6 & 11 & 34 & 76 & 15 & 231 \\
\hline
\end{tabular}

The Pulkovo Spectrophotometric Catalog published by Alekseeva et al. (1996, 1997 ) should also be mentioned among the most representative and precise spectrophotometric catalogues.

The Asiago Database of Spectroscopic Databases (Sordo \& Munari, 2006) contains some 300 atlases of observed stellar spectra. However, the majority of them provide data for small numbers of stars or/and for a restricted spectral range. The most representative atlases are listed in Table (together with some earlier atlases) and shown in Fig. 7.

It should be noted that data presented in various atlases often significantly differ even for bright stars, especially in the UV range (see, for instance, Fig. 8).

Synthetic magnitudes and colors can be calculated for stars presented in empir- 


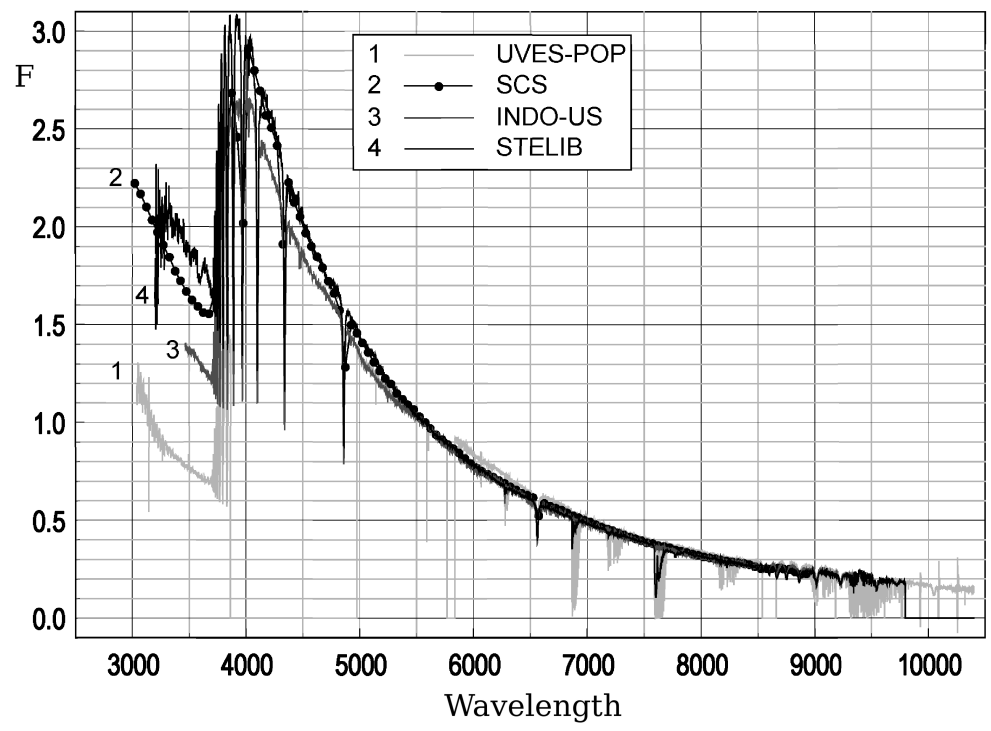

Fig. 8. The spectral energy distribution of $\alpha$ Leo, as presented in different atlases. The curves are normalized at $5500 \AA$. Note a significant disagreement in the UV range.

ical atlases by combining their spectral energy distributions with response curves. Such magnitudes and colors in the $W B V R$ system were calculated for stars catalogued in Kornilov et al. (1991) and included in the atlases. The results of our comparison for several spectrophotometric atlases are presented in Table .

Our preliminary analysis of modern empirical spectral atlases shows the following.

- Accuracy of the ground-based SSS and of the space-born NGSL atlases is comparable. The standard error of synthetic photometry, calculated from the two SEDs, reaches $0^{m} .02$.

- The UVES-POP atlas is precise enough; however, sometimes different spectral ranges are not sewed accurately.

- Systematic errors of the other atlases are significant (in excess of $0^{m} .1$ ).

- Spectrum calibration problems in the UV spectral range remain unsolved.

\section{CONCLUSIONS}

We have developed a method for reliable cross-matching of large multi-wavelength catalogues with different object densities and constructed a tool for simulation of color-index diagrams. The constructed diagrams use combined photometry, obtained from cross-matched catalogues, and can serve for parameterization of single and, when possible, binary stars.

The method was tested for the most representative modern catalogues. It was shown that GALEX/SDSS photometry can be used for (even reddened) singlebinary star separation and for parameterization of stars. Beside stellar astrophysical parameters, interstellar extinction can be determined in a given area of the 
sky, and thus a 3D galactic interstellar extinction map can be constructed. This problem remains topical: there are hundreds of articles about distribution of the reddening material in the Galaxy (e.g., cf. the catalogue by Burnashev et al. 2012); however, a misfit of some results means there is a lot of work to be done.

The method can be applied, in particular, to coming space-borne catalogues: Gaia (http://www.esa.int/science/gaia), Lyra-B (Mironov et al. 2010), WSO-UV (Shustov et al. 2011; http://wso.inasan.ru/), Svecha (Prokhorov et al. 2009).

We have also found that significant corrections should be made to modern empirical and semi-empirical stellar spectral atlases in order to use their data for calculating synthetic stellar magnitudes. Construction of a combined stellar atlas could be a challenging task.

ACKNOWLEDGMENTS. The authors thank Marina Lobanova and Veta Avedisova for collaboration and valuable comments. This work has been supported by Russian Foundation for Fundamental Research grants 09-02-00520, 10-07-00342 and 10-02-00426, by the Federal Science and Innovations Agency under contract 02.740.11.0247, by the Federal target-oriented program "Scientific and pedagogical staff for innovation Russia" (contract No. P1195), and by the program 4354.2008.2 "Leading Scientific Schools Support" of the Presiduum of Russian Academy of Sciences. A.K. acknowledges support from the National Research Foundation of South Africa. This research has made use of the SIMBAD and VizieR databases, operated at the Centre de Données astronomiques de Strasbourg, and of NASA's Astrophysics Data System Bibliographic Services.

\section{REFERENCES}

Alekseeva G. A., Arkharov A. A., Galkin V. D. et al. 1996, Baltic Astronomy, 5, 603 ;

1997, Baltic Astronomy, 6, 481

Bagnulo S., Jehin E., Ledoux C. et al. 2003, Messenger, 114, 10

Breger M. 1976, ApJS, 32, 7

Budavari T., Heinis S., Szalay A. S. et al. 2009, ApJ, 694, 1281

Burnashev V. I., Grigorieva E. A., Malkov O. Yu. 2012, Baltic Astronomy, submitted

Falcon-Barroso J., Sanchez-Blazquez P., Vazdekis A. et al. 2011, arxiv:1107.2303v1

Fluks M. A., Plez B., The P. S. et al. 1994, A\&AS, 105, 311

Glushneva I. N., Doroshenko V. T., Fetisova T. S. et al. 1980a, Soobshch. Gos. Astron. Inst. P.K. Shternberga, 219, 3; 1980b, Astron. Zh., 57, 1003;

1982, Trudy Gos. Astron. Inst. Shternberga, 52, 182

1983, Trudy Gos. Astron. Inst. Shternberga, 55, 84

1989, Trudy Gos. Astron. Inst. Shternberga, 61, 272

1991, Trudy Gos. Astron. Inst. Shternberga, 62, 119

Glushneva I. N., Doroshenko V. T., Fetisova T. S. et al. 1982, Spectrophotometry of Bright Stars, ed. Glushneva I. N., Moscow, Nauka, p. 3-252;

1983, Trudy Gos. Astron. Inst. Shternberga, 53, 50

1984, Trudy Gos. Astron. Inst. Shternberga, 54, 3

Glushneva I. N., Kharitonov A. V., Kniazeva L. N, Shenavrin V. I. 1992, A\&AS, 92,1

Gunn J. E., Stryker L. L. 1983, ApJS, 52, 121

Gunn J. E., Carr M., Rockosi C. et al. 1998, AJ, 116, 3040 
Heap S. R., Lindler D. J. 2007, ASPC, 374, 409

Karpov S. V., Mironov A. V., Malkov O. Yu. 2012, Astrophysical Bulletin, in press

Kharitonov A. V., Tereshchenko V. M., Knyazeva L. N. 1988, Alma-Ata, Nauka, p. 484 ;

2011, Spectrophotometric Catalogue of Stars, ed. Tereshchenko V. M., AlmaAty, Kazak University, p. 4-304

Kornilov V. G., Volkov I. M., Zakharov A. I. et al. 1991, Catalogue of WBVR magnitudes of Bright Northern Stars, Ed. Kornilov V. G., Moscow State University Publishers

Le Borgne J.-F., Bruzual G., Pello R. et al. 2003, A\&A, 402, 433

Malkov O. Yu., Karpov S. V., 2011, Cross-matching large photometric catalogs for parameterization of single and binary stars, in ADASS XX Conference, eds. I. N. Evans et al., ASPC, 442, p. 583

Malkov O. Yu., Sichevskij S. G., Kovaleva D. A. 2010, MNRAS, 401, 695

Malkov O. Yu., Mironov A. V., Sichevskij S. G. 2011, Ap\&SS, 335, 105

Mironov A. V., Zakharov A. I., Prokhorov M. E. et al. 2010, in Variable Stars, the Galactic halo and Galaxy Formation, eds. C. Sterken, N. Samus, L. Szabados, Zvenigorod, Published by Sternberg Astronomical Institute of Moscow University, Russia, p. 185

Morrissey P., Schiminovich D., Barlow T. A. et al. 2005, ApJ, 619, L7

Pickles A. J. 1998, PASP, 110, 863

Prokhorov M., Zakharov A., Mironov A. 2009, Space astro-photometric experiment Svecha, Proceedings of the Institute of Applied Astronomy, St. Petersburg, Russia, Nauka (in Russian), vol. 20, p. 336

Shustov B., Sachkov M., Gomez de Castro A. I. et al. 2011, Ap\&SS, 335, 273

Sichevskij S. G. 2012, Astronomy Reports, in press

Sichevskij S. G., Mironov A. V., Malkov O. Yu. 2012, Baltic Astronomy, submitted

Sordo R., Munari U. 2006, A\&A, 452, 735

Sviderskienè Z. 1988, Bull. Vilnius Obs., No. 80, 3

Valdes F., Gupta R., Rose J. A. et al. 2004, ApJS, 152, 251

Wu Y., Singh H. P., Prugniel P. et al. 2011, A\&A, 525, A71 